\title{
An Investigation on the Configuration of Spacesuit Soft Joints
}

\author{
Qilin Liu, Xiangyang Liu \\ China Astronauts Research and Training Center, Beijing, China \\ liuqilin01024488@163.com
}

\begin{abstract}
The mobility joint is of critical importance for the spacesuit to move from place to place. Especially, the joint configuration has a significant impact on spacesuit flexibility. This paper classifies the most commonly used soft joints of spacesuits into three types, namely for convolute joints, wrinkling joints and hybrid joints, based on the structure features. Sequentially, the structure characteristics and abilities to maintain constant volume are analyzed for all types of joints.
\end{abstract}

Keywords-spacesuit, joint, convolute, wrinkling, hybrid

\section{INTRODUCTION}

In current extravehicular spacesuits, which are fully pressurized closed garments, flexibility is provided by placing joints at such locations as elbows, knees or shoulders. The spacesuit joints can be divided into hard joints and soft joints depending on the material. Usually, the soft one is crafted with flexible stuff of high tensile strength such as sewn fabric, rubber, polymer, etc.

For spacesuit soft joints, there are many different structure forms, which exist discrepancies in terms of activities performance and workmanship. To design spacesuits, it is essential to be familiar with joint structure forms. Gary L.Harris ${ }^{[1]}$ reported some qualitative features of traditional joint shapes, while he didn't depict common characters of them. As the research goes far, there are some other new soft joint structure forms like origami joints ${ }^{[2]}$ and isotensoid joints ${ }^{[3]}$ introduced by Kengo IKEMA and Anna V. The primary aim of this paper is to investigate the configuration of spacesuit soft joints and conclude general characters of them. Firstly, the thesis describes the source of spacesuit joint torque. And then, it classifies the most commonly used soft joints of spacesuits into three types, namely for convolute joints, wrinkling joints and hybrid joints, based on the design ideas. The structure forms and abilities to maintain constant volume are analyzed for all types of joints sequentially.

\section{The SOURCE OF SPACESUIT JOINT TORQUE}

In order to enhance spacesuit joint mobility, the torque should be decreased as possible. Therefore, it is necessary to comprehend causes of joint torque. From the point of view of energy, when the joint is driven by pure bending load, the potential energy is given as:

$$
\Pi=p V+M \theta+E_{z}
$$

Where $p$ is the pressure, $V$ is the internal volume, $M$ represents the bending moment, $\theta$ represents bending angle and $E_{z}$ represents strain energy of joint shell components.

According to the principle of minimum potential energy, the equation above can be transformed as:

$$
M=-\left(p \frac{d V}{d \theta}+\frac{d E_{z}}{d \theta}\right)
$$

It can be clearly observed from the expression that one part of the work done by the bending moment compresses joint internal volume ${ }^{[4]}$ and another part is converted to strain energy. In most cases, the volume change is the main source of joint resistance torque ${ }^{[5]}$. For this reason, it is a crucial factor that maintains a constant internal volume in the joint structure design. The following describes three types of joint structures and analyzes their properties to keep the volume unchanged.

\section{CONVOLUTE JOINTS}

The overall morphology of convolute joints, which are designed to match the geometric characteristics of human joints, is approximate revolution surface after the structures are pressurized. Usually, the minimum cross section is shaped by circumferential restriction elements during the motion of convolute joints. Hence, this type of joints can maintain a relative constant internal volume. The following are some typical convolute joints.

\section{A. The Bellows Joint}

The bellows joint is one of the most classic joint forms. As Fig. 1 shows, the circumferential restraining cables, whose length is a little shorter than the cross-sectional perimeter of the joint cylinder, are attached to the structure shell. Bellows units are formed by restrictions between adjacent circumferential cables. As the bending moment is exerted on the joint, one side of the bellows unit contracts while the other side expands. 


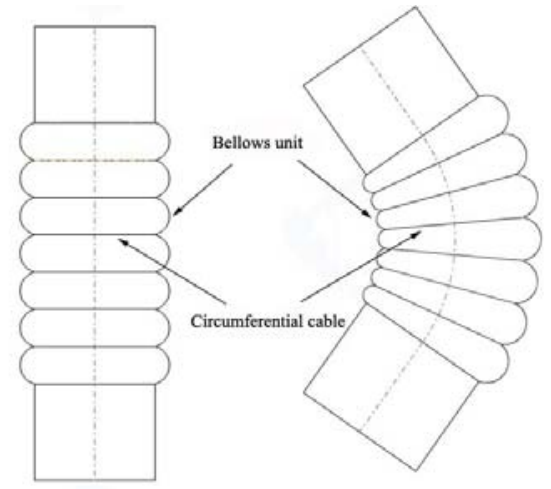

Figure 1. The Bellows Joint

There exists spring return phenomenon when the bellows joint is flexed. As William Elkins pointed out, when the center of restraint (CR) and the center of pressure (CP) were co-incident, it would flex throughout its bending range with minimal volume change and minimum bending torque for single-axis mobility joints ${ }^{[6]}$ (see Fig.2). However, with a flexed bellows joint, the compressed side of the joint bunch-up and are squeezed outward, and the tensile side flatten inward because of the flexibility of material. This phenomenon physically takes the CP away from the CR. Therefore, spring return moment occurs when the bellows joint bends.

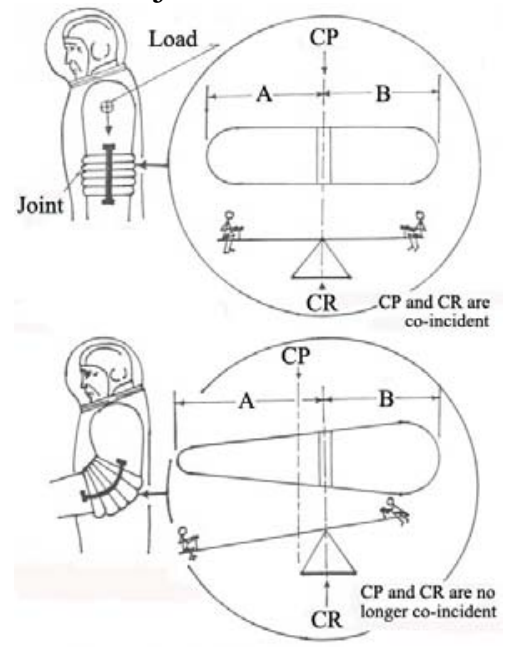

Figure 2. Spring Return Phenomenon

\section{B. The Toroidal Mobility Joint}

The toroidal mobility joint, to put it in the simplest technical terms, is a fabric, single wall laminate ${ }^{[1]}$ convoluted mobility joint where convolutes (major diameter and minor diameter) are supported and shaped by rigid circumferential rings which are attached transversely (perpendicular at right angles) to the longitudinal axis of the joint. As Fig.3 shows, the external circumferential ring (ring 1) supports the shape of the major diameter, and the internal circumferential ring (ring 2) constricts the minor diameter.

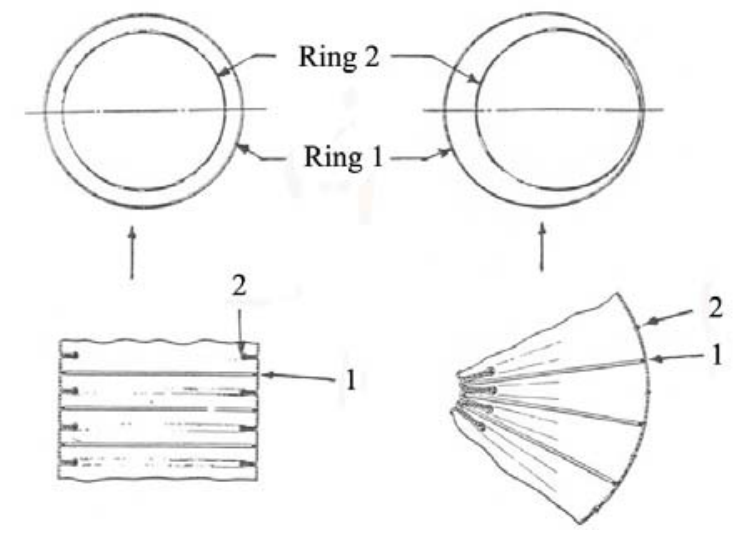

Figure 3. The Toroidal Mobility Joint

The engineering advantage of the toroidal mobility joint, over that of the bellows joint, is that the major diameter segment of the toroidal convolutes does not balloon-out as the joint is flexed, and the inner circle moves to a point of tangency with the outer circle. Thus, a toroidal mobility joint segment (major diameter) will not change its shape or grow away from the center of restraint on the compressed side of the joint when flexed. The CR and the $\mathrm{CP}$ are almost co-incident, which results in small volume change and low joint moment.

\section{Flat Panel Convolute}

The simplest type of mobility joint is the flat panel convolute $^{[7]}$. This fabric convolute joint is formed by a series of circumferential non-elastic fabric bands, sewn at even intervals around a long conical fabric tube. A longitudinal restraint strap, running down both sides of the conical tube (at the neutral axis), is passed through a retainer or guide on each circumferential band. The fabric has its weave laid 45 degrees bias of the longitudinal direction of the joint ${ }^{[1]}$.

When the flat panel convolute is flexed, there exists spring return phenomenon which is similar to that of the bellows joint. So the CR and CP would not co-incident. Spring return moment occurs when flat panel convolutes bend. 


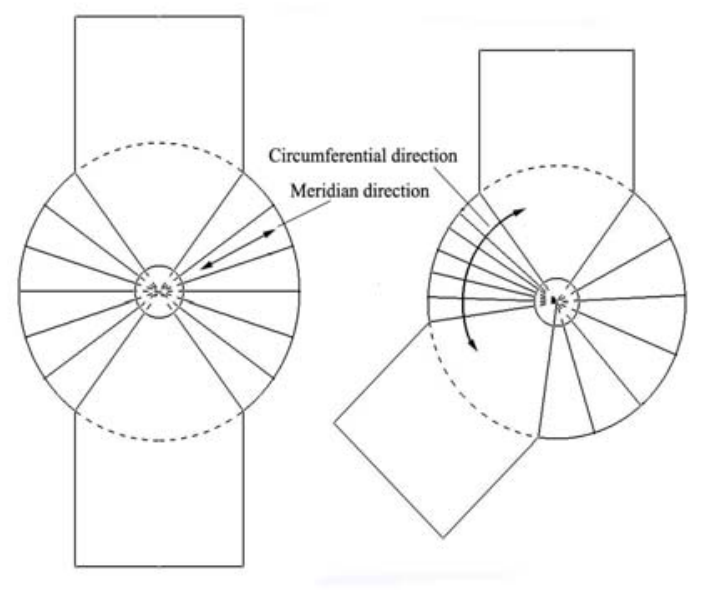

Figure 4. Flat Pattern Joint

\section{Dual Opposed Convolute}

This type of joint can be envisioned as a single slice of a bellows convolute that pivots on an internal rigid linkage. This linkage allows opposing sides of the joint fabric to expand or contract to maintain constant volume ${ }^{[1]}$, as the joint is flexed. The dual opposed joint has a very limited motion range, usually around 25 degrees of arc length, which restricts its application to areas not requiring high mobility, such as the ankle and waist area.

\section{WRINKLING JOINTS}

Wrinkling joints can be bent or stretched through the way that excess material is added to the structure to form wrinkles. On the tensile side of the joint, the folds balloon out and form a series of crude, hard to predict, semi-convolute shapes, when the joint is pressurized and flexed. On the compressed side of the joint, the folds "roll" over one another. Hence, wrinkling joints can maintain a relative constant internal volume. The following are two typical wrinkling joints.

\section{A. Flat Pattern Joint}

This type of joints can be made in many variations. The common one is made from a conical, flexible pressure bladder and restraint fabric. On the tensile side of the joint, the fabric of the restraint layer is "tucking" into a series of transverse patterns telescope fashion. And on the compressed side, the fabric is shaped as a semi-cone. The longitudinal restraining straps are stitched along both sides of the joint (along the neutral axis).

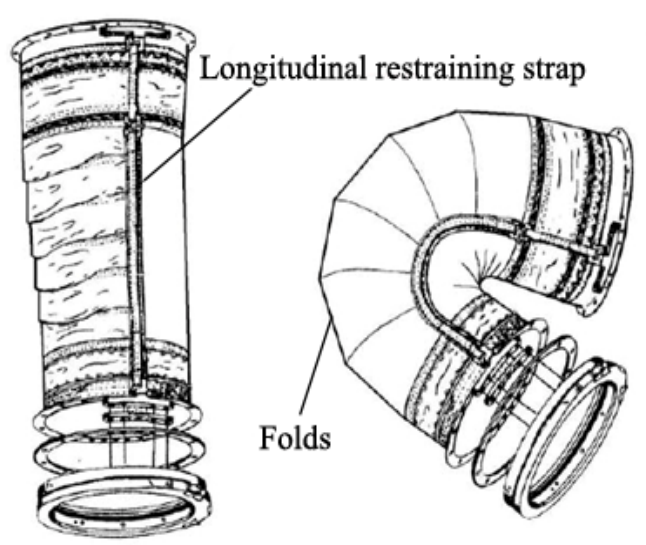

Figure 5. Isotensoid Joint

A drawback of flat pattern joint is that it is difficult to predict the exact area where the joint will break, i.e. the exact crease, on the compressed side of the joint, where the joint begins to bend. The flat pattern joint's neutral range is between 20 to 90 degrees, depending on its method of manufacture. In addition, the flat pattern joint forms a " $U$ " shape when deflected past 90 degrees. This tendency means the joint does not follow normal human anthropometry, and thus can exhibit high bending moments ${ }^{[8]}$.

\section{B. Isotensoid Joint}

This joint is based on the implementation of an isotensoid shape ${ }^{[9,10]}$ that can theoretically carry interior pressure without circumferential stress. By introducing excess skin material into the isotensoid along a circumferential direction, local slack regions are generated, which enable expansion and contraction, resulting in a flexion motion with low resistance torque ${ }^{[3]}$.

The inner volume change with flexion angle remains low up to 80 degrees, as extension on the outer side and contraction on the inner side occurred at the same rate. Fiber reorientation and buckling phenomena are considered to be the primary reasons for volume change beyond 80 degrees.

\section{HYBRID JOINTS}

Hybrid joints combine characteristics of convolute joints with those of wrinkling joints. Usually, the minimum cross section is shaped by circumferential restriction elements. And excess material is added to the structure to form wrinkles for enhancing joint mobility. The following are three representative hybrid joints.

\section{A. The Rolling Convolute}

The rolling convolute is made through the way that a cylinder of straight fabric and bladder material is foreshortened by “tucking in" deep circumferential folds ${ }^{[1]}$. These folds make the cylinder take on the appearance of a telescope. Into the folds are inserted, and bonded, 
aluminum bands. In turn, these bands are attached to an external exo-skeletal pivotal framework (a series of gimbal rings) that allows the joint to bend in two axes.

This joint does not form a series of bellows-like convolutions that can "bunch-up" or compress; rather it maintains constant volume because the arc radius on the tensile side of the joint lengthens to equal the arc radius on the compressed side. By this method the joint always retains its cylindrical shape, even when flexed, in which case it resembles a toroidal.

\section{B. Asymmetrical Semi-Toroid Joint}

Asymmetrical semi-toroid joint ${ }^{[11]}$ is a mix between a flat panel convolution and a true toroidal joint, using the best features of both to form a highly mobile, low-moment joint. A convolution is employed on the tensile side of the joint while a partial flat pattern fabric is used on the compressive side. Webbing tapes are bonded and sewn on circumferentially around the apex of the convolutions and in the minor diameter between each convolution. Then the longitudinal webbing restrain member is attached to the neutral areas at each convolute. The tape/restraint arrangement forces the joint, when it is flexed, to maintain co-incidence between the $\mathrm{CP}$ and the $\mathrm{CR}$ for much of its flexure range. Thus, the internal volume of this joint is nearly constant through much of its flexion range.

\section{Origami Joint}

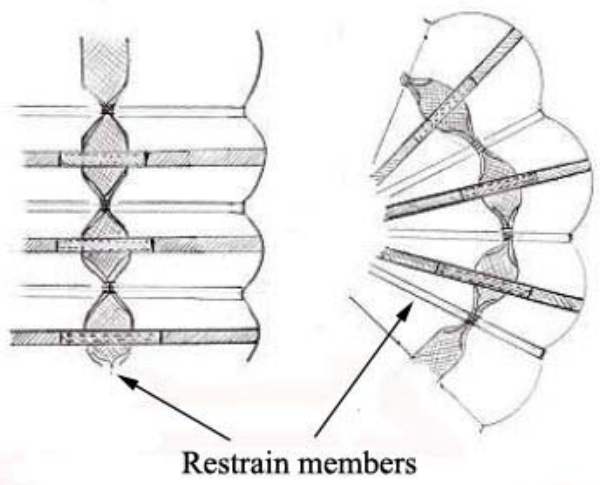

Figure 6. Asymmetrical Semi-Toroid Joint

The origami joint structure, Membrane-Beam Folding Structure $(\mathrm{MBFS})^{[2]}$, is proposed in the work to give flexibility to pneumatic tubes. MBFS represents a fabric tube with metal beams attached according to the Yoshimura folding pattern which theoretically enables to fold the shells without in-plane stretching when compressed.
The MBFS tube behaves like a spring with certain restoring force. Since frames three-dimensionally restrain tube's axial inflation, the MBFS tube becomes stable with certain length. At the same time, energy of internal pressure is stored into frames in the form of elastic strain energy which otherwise would work on fabric as prestress. Polygonal frames three-dimensionally restraint effect could maintain constant internal volume when the joint is flexed.

\section{CONCLUSION}

The most commonly used soft joints of spacesuits are classified into three types, namely for convolute joints, wrinkling joints and hybrid joints, based on the structure features. For each type of joints, some representative joints are introduced including structure characteristics and abilities to maintain constant volume to decrease joint torque. This classification, which helps us clearly understand the joint structural forms that may be used in the spacesuit design, is relatively qualitative. Not all the joint structures are listed in the paper. Thus, it can be supplemented furthermore

\section{REFERENCES}

[1] Gary L. Harris: The Origins and Technology of the Advanced Extravehicular Space Suit, volume 24 of AAS History Series, chapter, 1, American Astronautical Society Publishers (2001).

[2] Kengo IKEMA, Anna V: Deformation Analysis of a Joint Structure Designed for Space Suit with the Aid of an Origami Technology, JSASS Aerospace Tech Vol. 8, pp. Pp_1-Pp_5 (2010).

[3] Kengo IKEMA, Anna V: A Pneumatic Joint for Enhancement of Spacesuit Flexibility, submitted to Journal of Aerospace Engineering (2012).

[4] Fay J P, Steele C R: Bending and symmetric pinching of pressurized tubes, submitted to International Journal of Solids and Structures (2000).

[5] Holschuh B, Waldie J, Hoffman J, et al: Characterization of structural, volume and pressure components to space suit joint rigidity, submitted to 39th International Conference on Environ-mental Systems (2009)

[6] Elkins, William, Engineer: Life Support Systems Inc, Personal communication (1994).

[7] Pablo de Leon, Mark Williamson: The Development of a Planetary Suit Concept Demonstrator by the North Dakota Space Grant Consortium, submitted to SAE International (2006).

[8] Thomas K S, McMann H J: U S Spacesuits, Praxis Publishing (2006).

[9] Taylor, G.I: On the shapes of parachutes, The Scientific Papers of G.I.Taylor Vol. 3, pp. Pp_26-Pp_37 (1963).

[10] William H. Paulsen: What Is the Shape of a Mylar Balloon?,The American Mathematical Monthly, Vol. 101, No. 10, pp. Pp_953-Pp_958 (1994).

[11] Pablo de Leon, Gary L. Harris: NDX-2: Development of an Advanced Planetary Space Suit Demonstrator System for the Lunar Environment, submitted to 41st International Conference on Environmental Systems (2011). 